\title{
Upregulation of circRNA_0000285 serves as a prognostic biomarker for nasopharyngeal carcinoma and is involved in radiosensitivity
}

\author{
MINGXIA SHUAI, JANGWEI HONG, DONGHAI HUANG, XIN ZHANG and YONGQUAN TIAN \\ Department of Otolaryngology-Head and Neck Surgery, Xiangya Hospital, Central South University, \\ Changsha, Hunan 410008, P.R. China
}

Received January 17, 2018; Accepted July 30, 2018

DOI: $10.3892 / \mathrm{ol} .2018 .9471$

\begin{abstract}
Despite significant medical advancement, nasopharyngeal carcinoma (NPC) remains one of the most difficult types of cancer to detect and treat. Circular RNA (circRNA) signatures may be used as prognostic and predictive factors for cancer. Previous studies indicated that the biological role of circular homeodomain interacting protein kinase 3 (HIPK3) has cancer type-specificity. The HIPK3 gene locus formats three circRNA isoforms: circRNA_100783, circRNA_0000285 and circRNA_100782. However, their roles in NPC remain unknown. In the present study, whether these circRNAs could be used as a biomarker for NPC diagnosis and predicting treatment response was investigated. Reverse transcription-quantitative polymerase chain reaction was performed to measure the levels of circRNA_100783, circRNA_0000285 and circRNA_100782 in NPC and adjacent tissues. In addition, the circRNA_0000285 levels were further confirmed in serum samples from patients with NPC and healthy controls. The results demonstrated that circRNA_0000285, but not circRNA_100782 and circRNA_100783, was significantly increased in NPC tissues and serum samples from patients with NPC, compared with adjacent tissues and serum samples from healthy controls, respectively. Furthermore, circRNA_0000285 expression was increased in patients with radioresistant NPC, compared with patients with radiosensitive NPC. Further analysis demonstrated that circRNA_0000285 was significantly associated with tumor size $(\mathrm{P}<0.001)$, differentiation $(\mathrm{P}=0.022)$, lymph node metastasis $(\mathrm{P}=0.035)$, distant metastasis
\end{abstract}

Correspondence to: Professor Yongquan Tian, Department of Otolaryngology-Head and Neck Surgery, Xiangya Hospital, Central South University, 87 Xiangya Road, Changsha, Hunan 410008, P.R. China

E-mail: tianspringxy@163.com

Key words: nasopharyngeal carcinoma, circular homeodomain interacting protein kinase 3, circRNA_0000285, biomarker, radiosensitivity, circular RNA
$(\mathrm{P}=0.022)$ and Tumor-Node-Metastasis stage $(\mathrm{P}<0.001)$. Additionally, univariate and multivariate analyses indicated that circRNA_0000285 may be an independent prognostic factor for the outcome of patients with NPC. The present data indicated that circRNA_0000285 may be a novel biomarker for NPC and is involved in NPC radiosensitivity.

\section{Introduction}

Nasopharyngeal carcinoma (NPC), an epithelial malignancy, arises from the superior aspect of the pharyngeal mucosal space (1). NPC is primarily prevalent in Asian, with 60,000 new NPC cases being reported in 2015 in China, and is associated with a combination of a number of factors, including viral, environmental, and hereditary factors (2). Despite significant medical advancement, NPC remains one of the most difficult cancer types to detect and treat (1). Until recently, the potential pathways and underlying mechanism of NPC were not well known (3). The capability to characterize cancer genomes is now providing insights into the genesis and molecular underpinnings of this disease (4). Increasing evidence indicates that aberrantly-expressed non-coding RNAs (ncRNAs), including microRNAs (miRs), long ncRNAs and a number of circular RNAs (circRNAs), are responsible for cancer initiation and progression, including for NPC (5). In addition to ncRNA, circRNAs also have been demonstrated to code proteins (6). The deregulation of circRNAs can cause them to act either as tumor suppressors or oncogenes, controlling cell proliferation, migration and metastasis (7). Furthermore, circRNA expression has been associated with radioresistant phenotypes in esophageal cancer cells (8). In addition, circRNA signatures may be used as prognostic and predictive factors for specific cancer types, including gastric cancer and glioma, potentially offering a clinical tool for assessing the disease state, as well as predicting the treatment response and clinical outcome (9). These results highlight the importance of circRNAs in mediating oncogenic processes (10); therefore, further examination and validation of the role of deregulated circRNAs in NPC will provide insight into the fundamental drivers of this disease.

Circular homeodomain interacting protein kinase 3 (circHIPK3) is an abundant circRNA produced from the 
HIPK3 gene and formatting the circle through the long intronic complementary repeat elements (10). Silencing of circHIPK3 inhibits the proliferation of HuH-7, HCT-116 and HeLa cells (11); however, circHIPK3 expression is associated with progression (12), and functions as a tumor suppressor in bladder cancer by sponging miR-558 (13). Thus, the biological role of circHIPK3 would be specific in different types of cancer. Through circBase (www.circbase.org), the HIPK3 gene locus was determined to format the circRNA isoforms circRNA_100783, circRNA_0000285 and circRNA_100782 (Table I). Their roles in NPC remain unknown; therefore, in the present study, whether these circRNAs could be used as a biomarker for NPC diagnosis and predicting the treatment response was investigated.

\section{Materials and methods}

NPC cancer tissue and serum sample collection. A total of 150 NPC tissues (69 males and 81 females; age range, 36-78 years) and 100 adjacent tissues (45 males and 55 females; age range, 32-75 years) were collected from patients who underwent biopsy or tumorectomy at the Department of Otolaryngology Head and Neck Surgery, Xiangya Hospital (Changsha, China) between September 2009 and October 2017. These patients did not receive radiotherapy or chemotherapy prior to surgery. The serum samples, including 100 healthy patients (from physical examinations at Xiangya Hospital) and 150 patients with NPC were also collected from patients who underwent radiotherapy and/or cisplatin-based chemotherapy at the Department of Otolaryngology Head and Neck Surgery, Xiangya Hospital between September 2010 and October 2017. Among these patients, 25 cases received only cisplatin-based chemotherapy. The medical records of patients with NPC with clinical Tumor-Node-Metastasis (TNM) staging (14) and survival information were collected. Patients with radioresistant NPC were defined as those with persistent disease $>6$ weeks, and those with recurrent disease $>2$ months after completion of radiotherapy. Patients with radiosensitive NPC were defined as those without local residual lesions at 6 weeks or no recurrence at 2 months after completion of radiotherapy. The present study was approved by the Ethic Committee of The Xiangya Hospital, Central South University (Changsha, China). All subjects provided written informed consent to participate in the present study.

Cell lines. The normal nasopharyngal epithelial cells NP69 and 4 NPC cell lines (HK-1, C666-1, 5-8F and SUNE-1) were obtained from Cellbank of Chinese Academy of Sciences (Shanghai, China). Cells were grown routinely in RPMI-1640 medium (Invitrogen; Thermo Fisher Scientific, Inc., Waltham, MA, USA) supplemented with $10 \%$ fetal bovine serum (Gibco; Thermo Fisher Scientific, Inc.) and cultured in a $37^{\circ} \mathrm{C}$ humidified atmosphere containing $5 \% \mathrm{CO}_{2}$.

Reverse transcription-quantitative polymerase chain reaction (RT-qPCR) analysis. TRIzol ${ }^{\circledR}$ reagent (Invitrogen; Thermo Fisher Scientific, Inc.) was used to extract total RNA from cells, tissue and serum samples, according to manufacturer's instruction. An ABScript II complementary DNA (cDNA) First-Strand Synthesis kit (cat no. RK20400;

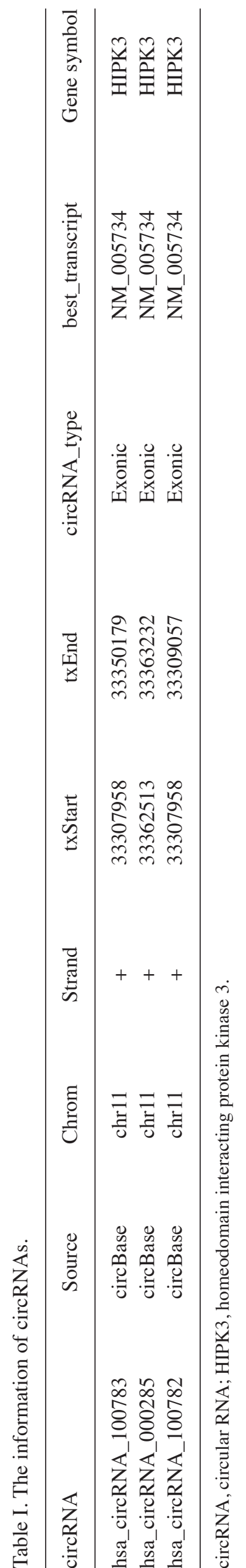



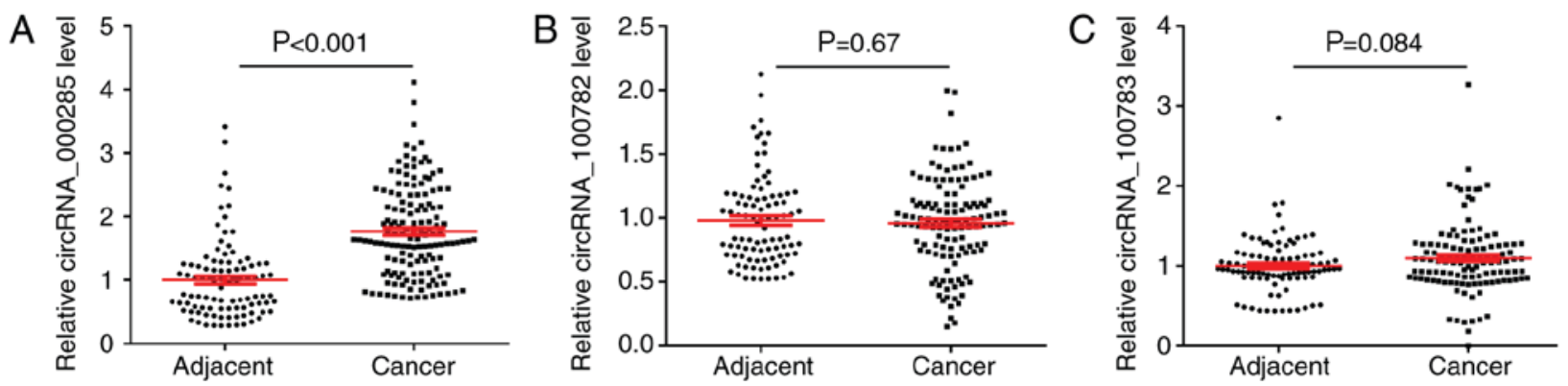

Figure 1. The circRNAs expression in NPC and adjacent tissues. Reverse transcription-quantitative polymerase chain reaction was performed to measure the expression of (A) circRNA_0000285, (B) circRNA_100783 and (C) circRNA_100782 in NPC ( $\mathrm{n}=150)$ and adjacent tissues (n=100). circRNA, circular RNA; NPC, nasopharyngeal carcinoma.

ABclonal Biotech Co., Ltd, Wuhan, China) was used to reverse transcribe cDNA from $500 \mathrm{ng}$ RNA, according to the manufacturer's protocols. The sequence of circRNA results were acquired from the database circBase. The expression of circRNAs was measured by SsoFast ${ }^{\mathrm{TM}}$ EvaGreen $^{\circledR}$ supermix (cat no. 1725201; Bio-Rad Laboratories, Inc., Hercules, CA, USA), according to manufacturer's protocols. Expression of $\beta$-actin was used as an endogenous control. qPCR was performed with the thermocycling conditions: $95^{\circ} \mathrm{C}$ for $3 \mathrm{~min}$, and 39 cycles of $95^{\circ} \mathrm{C}$ for $10 \mathrm{sec}$ and $60^{\circ} \mathrm{C}$ for $30 \mathrm{sec}$. The primers were as follows: circRNA_000285, forward 5'-TATGTTGGTGGATCCTGTTCGGCA-3', and reverse, 5'-TGGTGGGTAGACCAAGACTTGTGA-3'; circRNA_100782, forward, 5'-TATGTTGGTGGATCC TGTTCGGCA-3', and reverse, 5'-TGGTGGGTAGACCAA GACTTGTGA-3'; circRNA_100783, forward, 5'-GCTGCG GCCTGACTCTAAG-3', and reverse, 5'-TGTGCTTGATGG TGTCCCT-3'; and $\beta$-actin, forward, 5'-TTGTTACAGGAA GTCCCTTGCC-3', and reverse, 5'-ATGCTATCACCTCCC CTGTGTG-3'. The data were analyzed via the comparative threshold cycle $\left(2^{-\Delta \mathrm{Cq}},-\Delta \mathrm{Cq}=\mathrm{Cq}_{\text {circ }}-\mathrm{Cq}_{\beta \text {-actin }}\right)$ method, as previously described (15).

Statistical analysis. All data from three independent experiments were expressed as the mean \pm standard deviation and processed using SPSS 17.0 statistical software (SPSS, Inc., Chicago, IL, UA). The overall survival rate estimates over time were calculated using the Kaplan-Meier method with log-rank test. The clinical association between circRNA_000285 expression and clinicopathological variables in patients with NPC was evaluated by $\chi^{2}$ test. The difference among the groups was calculated by Student's t-test or one-way analysis of variance with Turkey post-hoc test, depending on the conditions. $\mathrm{P}<0.05$ was considered to indicate a statistically significant difference.

\section{Results}

circRNA_000285 is upregulated in NPC tissues and cell lines. RT-qPCR was performed to measure the expression of three circRNAs derived from HIPK3, circRNA_000285, circRNA_100782 and circRNA_100783, in 150 NPC and 100 adjacent tissues. It was determined that circRNA_000285 was significantly increased in NPC tissues ( $\mathrm{P}=0.0007)$, compared with the adjacent control (Fig. 1A), whereas the expression of

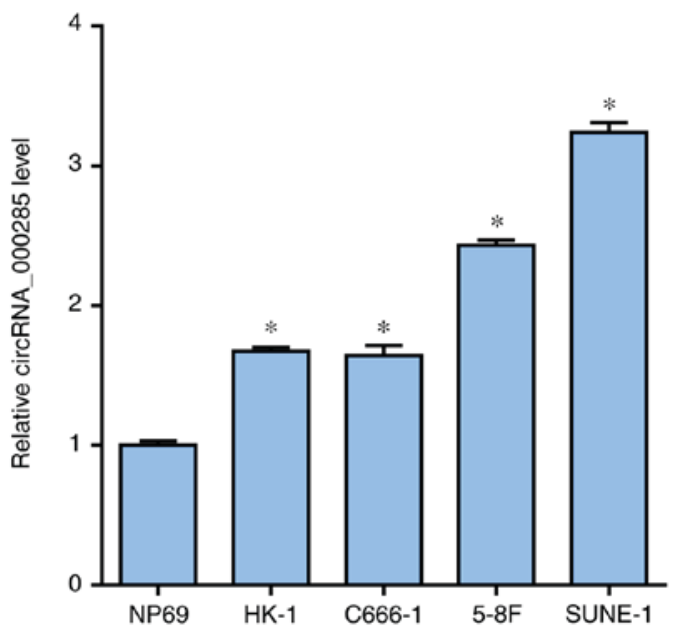

Figure 2. The expression of circRNA_0000285 in NPC cancer cells. Reverse transcription-quantitative polymerase chain reaction was performed to measure the expression of circRNA_0000285 in four NPC cancer cell lines and the normal nasopharyngal epithelial NP69 cell line. ${ }^{*} \mathrm{P}<0.05$ vs. NP69. circRNA, circular RNA; NPC, nasopharyngeal carcinoma.

circRNA_100782 and circRNA_100783 was not statistically significant between the NPC and adjacent tissues (Fig. 1B and C). Additionally, the expression of circRNA_000285 was also measured in four NPC cell lines, and it was observed that circRNA_000285 was significantly increased in these NPC cell lines $(\mathrm{P}=0.032$ for $\mathrm{HK}-1 ; \mathrm{P}=0.021$ for $\mathrm{C} 666-1 ; \mathrm{P}=0.014$ for $5-8 \mathrm{~F}$; and $\mathrm{P}=0.008$ for SUNE-1), compared with the normal nasopharyngeal epithelial NP69 cells (Fig. 2).

circRNA_000285 is upregulated in serum from patients with NPC. To investigate whether circRNA_000285 is a beneficial non-invasive biomarker for NPC, RT-qPCR was performed to detect the expression of circRNA_000285 in an independent cohort, including 150 serum samples of patients with NPC and 100 serum samples of healthy patients. circRNA_000285 was also significantly upregulated in the serum samples of patients with NPC, compared with the samples of healthy patients ( $\mathrm{P}=0.0005$, Fig. 3A). Notably, the circRNA_000285 level was significantly increased by 3 -fold in patients with radiation resistant NPC $(n=45)$, compared with patients with radiation sensitive NPC $(n=80)(P=0003$, Fig. 3B), indicating that circRNA_000285 may be a biomarker for NPC diagnosis and radiotherapy response. 
Table II. Clinical association between serum circRNA_000285 levels and clinicopathological variables of patients with nasopharyngeal carcinoma.

Serum circRNA_000285

\begin{tabular}{|c|c|c|c|}
\hline Variables & Low expression $(n=45)$ & High expression $(n=105)$ & $\chi^{2}$ test P-value \\
\hline Age & & & 0.533 \\
\hline$<50$ & 12 & 23 & \\
\hline$\geq 50$ & 33 & 82 & \\
\hline Sex & & & 0.99 \\
\hline Male & 21 & 48 & \\
\hline Female & 24 & 57 & \\
\hline Tumor size (longest diameter) & & & $<0.001$ \\
\hline$<3 \mathrm{~cm}$ & 28 & 32 & \\
\hline$\geq 3 \mathrm{~cm}$ & 17 & 73 & \\
\hline Differentiation $^{\mathrm{a}}$ & & & 0.022 \\
\hline High & 20 & 26 & \\
\hline Moderate & 15 & 34 & \\
\hline Low & 10 & 45 & \\
\hline Lymph node metastasis & & & 0.035 \\
\hline N0-1 & 26 & 41 & \\
\hline N2-4 & 19 & 64 & \\
\hline Distant metastasis & & & 0.022 \\
\hline No & 32 & 48 & \\
\hline Yes & 13 & 47 & \\
\hline TNM stage ${ }^{\mathrm{b}}$ & & & $<0.001$ \\
\hline I-II & 35 & 45 & \\
\hline III-IV & 10 & 60 & \\
\hline
\end{tabular}

circRNAs, circular RNA; TNM, Tumor-Node-Metastasis. ${ }^{a}$ Differentiation staging relies on American Joint Committee on Cancer (14); ${ }^{\text {bTNM }}$ staging relies on American Joint Committee on Cancer (14).
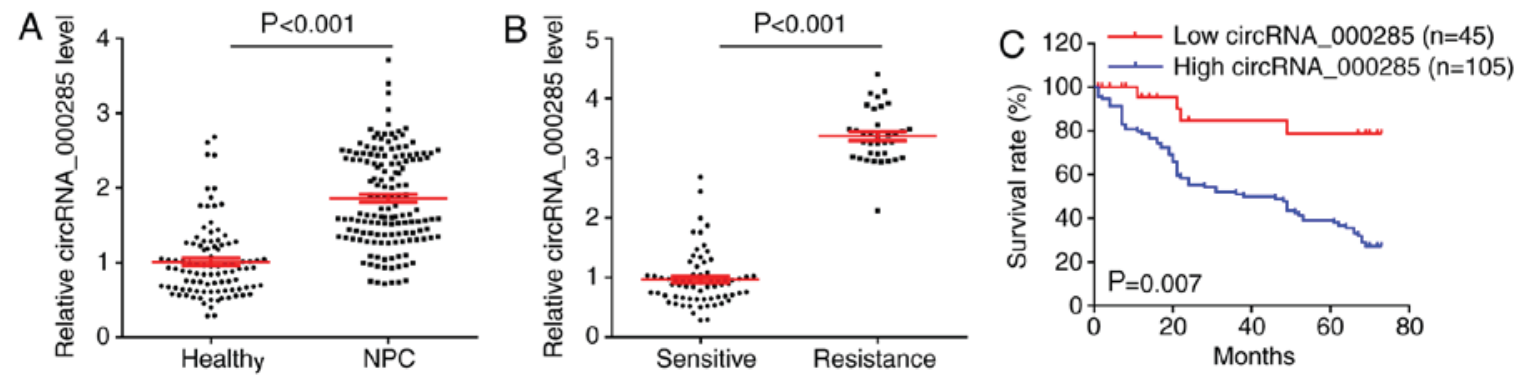

Figure 3. The expression of circRNA_0000285 in serum samples. (A) Reverse transcription-quantitative polymerase chain reaction was performed to measure the expression of circRNA_0000285 in serum samples of patients with NPC $(n=150)$ and healthy patients $(n=100)$. (B) The expression of circRNA_0000285 in serum samples of patients with radiosensitive NPC $(n=80)$ and radioresistant NPC $(n=45)$. (C) The Kaplan-Meier survival curve depicts the survival rate in patients with high $(\mathrm{n}=105)$ or low circRNA_000285 expression $(\mathrm{n}=45)$. circRNA, circular RNA; NPC, nasopharyngeal carcinoma.

circRNA_000285 is associated with pathoclinical features of patients with NPC. The association between circRNA_000285 and pathoclinical features of NPC cancer was also analyzed. The cases of NPC were divided into high circRNA_000285 expression, which was set as greater than the mean of circRNA_000285 levels, and low circRNA_000285 expression, which was set as lower than or equal to the mean of circRNA_000285 levels, groups. It was determined that circRNA_000285 expression was significantly associated with tumor size $(\mathrm{P}=0.0008)$, differentiation $(\mathrm{P}=0.022)$, lymph node metastasis $(\mathrm{P}=0.035)$, distant metastasis $(\mathrm{P}=0.022)$ and TNM stage $(\mathrm{P}=0.0007)$ (Table II). Additionally, the factors that could predict the prognosis of patients with NPC were investigated with univariate and 
Table III. Univariate analysis of prognostic factors of patients with nasopharyngeal carcinoma.

\begin{tabular}{lcc}
\hline Variables & Hazard ratio & P-value \\
\hline Age ( $\geq 50 /<50)$ & 1.08 & 0.08 \\
Sex (male/female) & 1.02 & 0.67 \\
Tumor size (longest & 2.65 & 0.02 \\
diameter; $\geq 3 \mathrm{~cm} /<3 \mathrm{~cm})$ & & \\
Differentiation ${ }^{\mathrm{a}}$ (low/high-moderate) & 2.84 & 0.03 \\
Lymph node metastasis (N2-4/N0-1) & 2.12 & 0.04 \\
Distant metastasis (yes/no) & 3.46 & 0.02 \\
TNM stage ${ }^{\mathrm{b}}$ (III-IV/I-II) & 2.67 & 0.03 \\
Serum circRNA_000285 levels & 3.28 & 0.01 \\
(high/low) & & \\
\hline
\end{tabular}

TNM, Tumor-Node-Metastasis; circRNAs, circular RNA. 'Differentiation staging relies on American Joint Committee on Cancer (14); ${ }^{\mathrm{b}} \mathrm{TNM}$ staging relies on American Joint Committee on Cancer (14).

multivariate analyses. Univariate analysis indicated that the serum circRNA_000285 level $(\mathrm{P}=0.01)$, as well as the tumor size $(\mathrm{P}=0.02)$, differentiation $(\mathrm{P}=0.03)$, lymph node metastasis $(\mathrm{P}=0.04)$, distant metastasis $(\mathrm{P}=0.02)$ and TNM stage $(\mathrm{P}=0.03)$ were significantly associated with the prognosis of patients with NPC (Table III). Multivariate analysis demonstrated that the serum circRNA_000285 level $(\mathrm{P}=0.01)$, tumor size $(\mathrm{P}=0.01)$, differentiation $(\mathrm{P}=0.03)$, lymph node metastasis $(\mathrm{P}=0.02)$, distant metastasis $(\mathrm{P}=0.01)$ and TNM stage $(\mathrm{P}=0.02)$ were independent factors for predicating the prognosis of patients with NPC (Table IV).

The association between serum circRNA_000285 levels and survival rate in patients with NPC was further analyzed. The Kaplan-Meier survival curve demonstrated that the patients with a high circRNA_000285 expression had a significantly decreased overall survival rate compared with those with a low circRNA_000285 expression ( $\mathrm{P}=0.007$; Fig. 3C). Therefore, the results indicated that circRNA_000285 serves a critical role in the development of NPC.

\section{Discussion}

In the present study, it was determined that circRNA_0000285, but not circRNA_100782 and circRNA_100783, was significantly increased in NPC tissues and serum of patients with NPC, compared with adjacent tissues and serum of healthy patients, respectively. Additionally, the circRNA_0000285 expression was significantly increased in patients with radioresistant NPC, compared with patients with radiosensitive NPC. Further analysis demonstrated that circRNA_0000285 could be an independent prognostic factor for the outcome of patients with NPC.

Emerging evidence revealed the function of circRNAs in cancer and that it may serve as a required novel biomarker and therapeutic target for cancer treatment $(16,17)$. Luo et al $(18)$ determined that aberrant circRNA_0000064 expression was associated with $\mathrm{T}$ stage, lymphatic metastasis and TNM stage in lung cancer. Additionally, it was previously indicated that
Table IV. Multivariate analysis of independent prognostic factors of patients with nasopharyngeal carcinoma.

\begin{tabular}{lcc}
\hline Variables $^{\mathrm{a}}$ & Hazard ratio & P-value \\
\hline Tumor size & 2.06 & 0.01 \\
Differentiation & 2.36 & 0.03 \\
Lymph node metastasis & 2.59 & 0.02 \\
Distant metastasis & 3.08 & 0.01 \\
TNM stage & 2.84 & 0.02 \\
Serum circRNA_000285 levels & 3.03 & 0.02 \\
\hline
\end{tabular}

${ }^{a}$ The cutoffs of these variables are identical to Table III. TNM, Tumor-Node-Metastasis; circRNAs, circular RNA.

circRNA_0074362 levels are significantly downregulated in gastric cancer tissues, and its levels were associated with lymphatic metastasis (9). Furthermore, it was demonstrated that circRNA_0001785 plasma has increased diagnostic accuracy, compared with carcinoembryonic antigen and cancer antigen 15-3, and its expression is associated with histological grade, TNM stage and distant metastasis (19). It was also indicated that circRNA_0001785 plasma level in postoperative patients was significantly reduced, compared with preoperative patients with breast cancer (19).

A previous study also determined that the isoform of hsa_circRNA_0000285, circRNA_100782, serves a role in other cancer types, including pancreatic carcinoma (20). Chen et al (20) determined that circRNA_100782 was notably upregulated in pancreatic ductal adenocarcinoma tissue. Additionally, downregulation of circRNA_100782 inhibited BxPC3 cell proliferation and colony formation by downregulating the miR-124 target genes interleukin-6 receptor and signal transducer and activator of transcription 3 (STAT3) (20). These data indicated that the functions of circRNAs are cancer-specific.

In its late stage, NPC is a refractory disease with a 2-year survival rate of $<50 \%$ (Hong Kong, 1976-1985) (21). However, if the patients with NPC were diagnosed in early stages, their 5-year survival rate would be $>80 \%$ due to the high radiosensitivity of NPC (Hong Kong, 1996-2000) (21). Radioresistance remains a notable obstacle for achieving successful treatment and causes local recurrence and distant metastases (22). Thus, emphasis has been placed on determining biomarkers and targets for radiotherapy. ncRNAs have been determined to be promoters or inhibitors of NPC radiotherapy (23). For example, upregulation of the lncRNA ANRIL attenuated the radiosensitivity in NPC by functioning as a miR-125a sponge (24). Additionally, miR-24 increases NPC radiosensitivity by directly regulating Jab1/CSN5 (25). In the present study, it was demonstrated that circRNA_0000285 expression is increased in patients with radioresistant NPC, compared with patients with radiosensitive NPC, and is increased in NPC cell lines, particularly in SUNE-1 cells that exhibit a high ability of metastasis, compared with normal epithelial cells. Thus, circRNA_0000285 may be a predictive biomarker of the radiotherapy response. miR-124 was reported as a tumor 
suppressor in NPC by targeting STAT3, calpain small subunit 1 and forkhead box Q1 (26-28). Additionally, miR-124 was a direct target of circRNA_100782 and circHIPK3 $(10,19)$. Silencing circHIPK3 inhibited cell proliferation and migration by increasing miR-124 and downregulating aquaporin 3 expression in hepatocellular carcinoma (29). circHIPK3 effectively inhibited migration, invasion and angiogenesis of bladder cancer cells by sponging miR-558 to suppress the expression of heparanase (13). These data indicated a possible mechanism of circRNA_0000285 regulation of NPC cell proliferation, metastasis and radiosensitivity.

To conclude, it was demonstrated that circRNA_0000285 was significantly upregulated in NPC tissues and serum. Additionally, the present study indicated that circRNA_0000285 may serve as a novel biomarker for NPC and is associated with NPC radiosensitivity.

\section{Acknowledgements}

Not applicable.

\section{Funding}

No funding was received.

\section{Availability of data and materials}

All data generated or analyzed during this study are included in this published article.

\section{Authors' contributions}

MS and YT made substantial contributions to the design of the study. JH, DH and XZ analyzed and interpreted the patient data. $\mathrm{JH}, \mathrm{DH}$ and MS performed cell biological experiments. All authors contributed to writing the manuscript. All authors read and approved the final manuscript.

\section{Ethics approval and consent to participate}

The present study was approved by the Ethic Committee of The Xiangya Hospital, Central South University (Changsha, China). All subjects provided written informed consent to participate in the present study.

\section{Patient consent for publication}

All patients provided written informed consent to participate in the present study.

\section{Competing interests}

The authors declare that they have no competing interests.

\section{References}

1. Bruce JP, Yip K, Bratman SV, Ito E and Liu FF: Nasopharyngeal Cancer: Molecular landscape. J Clin Oncol 33: 3346-3355, 2015.

2. He R, Hu Z, Wang Q, Luo W, Li J, Duan L, Zhu YS and Luo DX The role of long non-coding RNAs in nasopharyngeal carcinoma: As systemic review. Oncotarget 8: 16075-16083, 2017.
3. Lee AW, Poon YF, Foo W, Law SC, Cheung FK, Chan DK, Tung SY, Thaw M and Ho JH: Retrospective analysis of 5037 patients with nasopharyngeal carcinoma treated during 1976-1985: Overall survival and patterns of failure. Int J Radiat Oncol Biol Phys 23: 261-270, 1992.

4. Spence T, Bruce J, Yip KW and Liu FF: MicroRNAs in nasopharyngeal carcinoma. Chin Clin Oncol 5: 17, 2016.

5. Lee KT, Tan JK, Lam AK and Gan SY: MicroRNAs serving as potential biomarkers and therapeutic targets in nasopharyngeal carcinoma: A critical review. Crit Rev Oncol Hematol 103: 1-9, 2016.

6. Yang Y, Gao X, Zhang M, Yan S, Sun C, Xiao F, Huang N, Yang X, Zhao K, Zhou H, et al: Novel role of FBXW7 circular RNA in repressing glioma tumorigenesis. J Natl Cancer Inst 110: 2018.

7. Zhao J, Li L, Wang Q, Han H, Zhan Q and Xu M: CircRNA expression profile in early-stage lung adenocarcinoma patients. Cell Physiol Biochem 44: 2138-2146, 2017.

8. Su H, Lin F, Deng X, Shen L, Fang Y, Fei Z, Zhao L, Zhang X, Pan H, Xie D, et al: Profiling and bioinformatics analyses reveal differential circular RNA expression in radioresistant esophageal cancer cells. J Transl Med 14: 225, 2016.

9. Xie Y, Shao Y, Sun W, Ye G, Zhang X, Xiao B and Guo J: Downregulated expression of hsa_circ_0074362 in gastric cancer and its potential diagnostic values. Biomark Med 12:11-20, 2018.

10. Patop IL and Kadener S: circRNAs in Cancer. Curr Opin Genet Dev 48: 121-127, 2018.

11. Zheng Q, Bao C, Guo W, Li S, Chen J, Chen B, Luo Y, Lyu D, Li Y, Shi G, et al: Circular RNA profiling reveals an abundant circHIPK 3 that regulates cell growth by sponging multiple miRNAs. Nat Commun 7: 11215, 2016.

12. Okholm T, Nielsen MM, Hamilton MP, Christensen LL, Vang S, Hedegaard J, Hansen TB, Kjems J, Dyrskjøt L and Pedersen JS: Circular RNA expression is abundant and correlated to aggressiveness in early-stage bladder cancer. NPJ Genom Med 2: 36, 2017.

13. Li Y, Zheng F, Xiao X, Xie F, Tao D, Huang C, Liu D, Wang M, Wang L, Zeng F and Jiang G: CircHIPK3 sponges miR-558 to suppress heparanase expression in bladder cancer cells. EMBO Rep 18: 1646-1659, 2017.

14. Edge SB and Compton CC: The American Joint Committee on Cancer: The 7th edition of the AJCC cancer staging manual and the future of TNM. Ann Surg Oncol 17: 1471-1474, 2010.

15. Wei C, Wu S, Li X, Wang Y, Ren R, Lai Y and Ye J: High expression of FER tyrosine kinase predicts poor prognosis in clear cell renal cell carcinoma. Oncol Lett 5: 473-478, 2013.

16. Han YN, Xia SQ, Zhang YY, Zheng JH and Li W: Circular RNAs: A novel type of biomarker and genetic tools in cancer. Oncotarget 8: 64551-64563, 2017.

17. Zhang Y, Liang W, Zhang P, Chen J, Qian H, Zhang X and Xu W: Circular RNAs: Emerging cancer biomarkers and targets. J Exp Clin Cancer Res 36: 152, 2017.

18. Luo YH, Zhu XZ, Huang KW, Zhang Q, Fan YX, Yan PW and Wen J: Emerging roles of circular RNA hsa_circ_0000064 in the proliferation and metastasis of lung cancer. Biomed Pharmacother 96: 892-898, 2017.

19. Yin WB, Yan MG, Fang X, Guo JJ, Xiong W and Zhang RP: Circulating circular RNA hsa_circ_0001785 acts as a diagnostic biomarker for breast cancer detection. Clin Chim Acta: Oct 16, 2017 (Epub ahead of print).

20. Chen G, Shi Y, Zhang Y and Sun J: CircRNA_100782 regulates pancreatic carcinoma proliferation through the IL6-STAT3 pathway. Onco Targets Ther 10: 5783-5794, 2017.

21. Zhang L, Yang L, Li JJ and Sun L: Potential use of nucleic acid-based agents in the sensitization of nasopharyngeal carcinoma to radiotherapy. Cancer Lett 323: 1-10, 2012.

22. Chen $\mathrm{W}$ and $\mathrm{Hu} \mathrm{GH}$ : Biomarkers for enhancing the radiosensitivity of nasopharyngeal carcinoma. Cancer Biol Med 12: 23-32, 2015.

23. Ni J, Bucci J, Chang L, Malouf D, Graham P and Li Y: Targeting MicroRNAs in prostate cancer radiotherapy. Theranostics 7: 3243-3259, 2017.

24. Hu X, Jiang H and Jiang X: Downregulation of lncRNA ANRIL inhibits proliferation, induces apoptosis, and enhances radiosensitivity in nasopharyngeal carcinoma cells through regulating miR-125a. Cancer Biol Ther 18: 331-338, 2017.

25. Wang S, Pan Y, Zhang R, Xu T, Wu W, Zhang R, Wang C, Huang H, Calin CA and Yang H and Claret FX: Hsa-miR-24-3p increases nasopharyngeal carcinoma radiosensitivity by targeting both the 3'UTR and 5'UTR of Jab1/CSN5. Oncogene 35: 6096-6108, 2016. 
26. $\mathrm{Hu} \mathrm{H}$, Wang $\mathrm{G}$ and $\mathrm{Li} \mathrm{C}$ : miR-124 suppresses proliferation and invasion of nasopharyngeal carcinoma cells through the Wnt/b-catenin signaling pathway by targeting Capn 4 . Onco Targets Ther 10: 2711-2720, 2017.

27. Xu S, Zhao N, Hui L, Song M, Miao ZW and Jiang XJ: MicroRNA-124-3p inhibits the growth and metastasis of nasopharyngeal carcinoma cells by targeting STAT3. Oncol Rep 35: $1385-1394,2016$

28. Peng XH, Huang HR, Lu J, Liu X, Zhao FP, Zhang B, Lin SX, Wang L, Chen HH, Xu X, et al: MiR-124 suppresses tumor growth and metastasis by targeting Foxq1 in nasopharyngeal carcinoma. Mol Cancer 13: 186, 2014.
29. Chen G, Shi Y, Liu M and Sun J: circHIPK3 regulates cell proliferation and migration by sponging miR-124 and regulating AQP3 expression in hepatocellular carcinoma. Cell Death Dis 9: $175,2018$.

cc (i) $\ominus$ This work is licensed under a Creative Commons EY NC ND Attribution-NonCommercial-NoDerivatives 4.0 International (CC BY-NC-ND 4.0) License. 